\title{
Advocating for community-based behavioural approaches within the combination HIV prevention framework: A critical literature review
}

\author{
Renee West \\ GMI Partnership \\ Tony Furlong \\ METRO Charity \\ Greg Ussher \\ METRO Charity \\ Mark Santos \\ Positive East \\ Karen Skipper \\ Spectra
}

Kisley Di Giuseppe ( $\nabla$ kisley.giuseppe.14@ucl.ac.uk)

University College London https://orcid.org/0000-0001-7085-8895

Research Article

Keywords:

Posted Date: January 21st, 2022

DOI: https://doi.org/10.21203/rs.3.rs-1245923/v1

License: (c) (1) This work is licensed under a Creative Commons Attribution 4.0 International License. Read Full License 


\begin{abstract}
Efforts within the combination HIV prevention in the UK have not been sufficiently broad enough to address important aspects of behavioural issues to the end of the HIV epidemic, while also neglecting the role of community-level interventions in developing a more sustainable approach. The restricted impact presented in biomedical interventions calls for the redesign of a more comprehensive preventative protocol, where psychosocial and behavioural strategies that target high-risk local communities are more strongly emphasised. A comprehensive review of empirical studies was undertaken to investigate the reliability of community-based HIV prevention practices, which have targeted behavioural change in demographics and contexts similar to those presented in London. Further recommendations were developed and directed at London's HIV and sexual health leading actors. The findings of this review have reinforced the argument for the need of community-based, culturally relevant programmes alongside and interweaving with biomedical approaches, and not solely biomedical-focused HIV prevention.
\end{abstract}

\title{
Introduction
}

UNAIDS Fast Track Approach of ending AIDS by 2030 centres around globally achieving zero new HIV infections, zero AIDS-related deaths and zero discrimination. London's HIV and sexual health leaders and planners have embraced the UNAIDS (1) combination HIV prevention model of simultaneous biomedical as well as structural and behavioural interventions to achieve the 2030 goal. Condom use is a representation of how the synergy of structural, biomedical and behavioural preventative approaches can work together. Condoms as a biomedical tool can reduce HIV transmission (2). However, to increase the effectiveness of condom use, structural (e.g., guaranteeing the availability of condoms while reducing stigma) and behavioural (e.g., increasing knowledge and awareness through counselling and mass media campaigns) interventions at individual and community levels must be also encouraged to ensure longterm success (3).

In the UK, combination HIV prevention efforts have been directed to condoms and PrEP use, HIV testing, and related to concepts such as Treatment as Prevention (TasP) (4). Despite these major actions, HIV still remains a challenge in the city. For instance, despite a variety of interventions specific to promote condom use, the London Gay Men's Sexual Health Survey in 2016 showed an increase of 60\% of condomless anal sex in Men who have Sex with Men (MSM; 5), compared to the $43 \%$ in 2000 (6). Furthermore, the Public Health England's (PHE) report showed that $47 \%$ of young people did not use condoms when having sex with a new partner, including 1 in 10 never ever using condoms (4). In addition to interventions to promote condom use, HIV testing has been considered fundamental in reducing onward HIV transmission. In 2016, the British HIV Association (BHIVA, 7) treatment guidelines recommended early treatment for all HIV-positive individuals. Prompt diagnosis speeds up the time between seroconversion and start of treatment, while increasing the proportion of those diagnosed having an undetectable viral load. Despite this, some key barriers remain. Lack of testing among women is still an issue in London with limited UK HIV prevention services tackling HIV rarely directed at this gender, highlighting overall lack of prevention and general poor mental health support linked to living with HIV (8). A further exploratory study (9) showed that the above challenges were not limited to women and that psychological barriers such as low perception of risk could potentially affect the acceptability of TasP among MSM and Black African and Minority Ethnic groups (BAME). In addition to these treatments, the World Health Organisation (WHO) has recommended the use of oral Pre-Exposure Prophylaxis (PrEP) as a highly effective preventative approach for HIV-negative individuals at risk before any potential HIV exposure (10 for a review). Despite the overall increase in PrEP knowledge and accessibility in the UK, it has been showed to be of limited use especially among BAME and women (11). Specifically, barriers to PrEP intake have been perceived due to different levels of stigma: individual (negative association with promiscuity); interpersonal (families as being judgmental); community (automatically associated with being HIV+); and structural (judgment from service-providers) (12).

It is evident that even if the combination prevention's efforts in the UK have gone a long way, some limitations are still contributing to render HIV a public health concern in London. In fact, the significant increment of condomless anal sex, gender disparities in HIV testing coverage, and the presence of barriers affecting the acceptability of both PrEP and TasP seem to result from over-relying primarily on biomedical interventions, while neglecting the interplay of behavioural and structural elements (13). This is concerning, considering the fundamental importance of understanding the role played by the cultural, social and psychological elements presented within specific community settings.

\section{Importance of community-based interventions in getting to zero HIV infections}

As stated, individuals' behavioural change remains a fundamental instrument in the support of HIV prevention. Consequently, clearer understanding of the role of community-level involvement in getting to zero HIV infections is required. Community-based HIV prevention interventions aim at supporting individuals at risk through health promotion services within defined-geographically communities. They provide education, counselling and mass media campaigning to help their members reduce the risks of infection (14). The goal is to create a perception of community ownership where individuals become agents of change. By providing the combination of multi-level components, such as structural-level with community-level interventions (15), community-based programmes highlight the "importance of changing social norms by using community opinion leaders, role models, and peer educators as intervention specialists" (16, p.62). Collins and colleagues (17) see the implementation of evidence-based behavioural strategies within community-level interventions as the opportunity to bridge the gap between communities in need and HIV prevention intervention research. This would thus increase the effectiveness of a preventative programme within high-risk environments. Previous systematic reviews and meta-analysis of community-based approaches have demonstrated their worth in increasing HIV and Sexually Transmitted Infections (STI) testing in the community where needed (18). Furthermore, they have contributed to the expansion of voluntary HIV testing and counselling in MSM, People Living with HIV (PLWHIV) and Female Sex Workers (SW) (19). Finally, their implementation has improved antiretroviral adherence in PLWHIV (15) and increased condom use in MSM (20). It is evident from this that community-based organisations are uniquely placed with an important role in getting to zero HIV infections.

\section{Importance of behavioural interventions in getting to zero HIV infections}

Page 2/11 
Despite recent advances in biomedical interventions, HIV prevention researchers recognise the importance for these to be supported by behavioural approaches (21). For the purpose of this current paper, behaviour change interventions are considered to range from individual-level interventions to reduce condomless sex by promoting HIV testing and counselling (e.g., traditional counselling, peer education and testing); small group-level interventions among groups most at risk (risk-reduction skills training and sexual education); and community-level interventions (community empowerment and mass media campaigns to widespread change-belief about social norms) $(20,22,23)$. According to Global HIV Prevention Working Group (24), behavioural HIV prevention interventions aim to promote individual knowledge and skills needed to avoid and negotiate risky behaviour. They also aim to achieve results both at social and structural levels, by decreasing any stigma attached to the individuals' sexuality, while influencing gender roles and creating more supportive legal, policy and environmental norms. In order to change behaviour, the intersection of vulnerability and risk must be addressed. Therefore, interventions need to be evidence-informed, tailored to the specific target group, and scaled-up with sufficient coverage and enough intensity for a sustained period of time. Past research has demonstrated the effectiveness of behavioural strategies such as counselling, individual and group programmes, peer mentoring (25, 20) and couple-focused preventative counselling (26) in different population groups: BAME, MSM, PLWHIV, SW, Injecting Drug Users (IDU), Individuals Convicted of Crime (ICC), Individuals of Transgender Experience (ITE) and young people $(21,24,27)$. It is evident from the above the fundamental role of behavioural interventions within the combination prevention model in getting to zero HIV infections.

\section{The current paper}

This paper is undertaken in collaboration with ??? ???, a consortium of three charities (???, ??? and ???). ??? provides community-based HIV and sexual health prevention and support services in London to address sexual health inequalities of people from MSM, BAME, migrant communities and people of trans experience. Throughout its 12-year history, ??? has delivered services including counselling, mentoring, health awareness, outreach, HIV testing and STI screening, face to face support, group work and PrEP interventions. Moreover, ??? challenges norms and recognises intersectionality in London by modelling and championing services. In recent years, due to new policy decisions, budgetary constraints and funders' priorities, ???'s activities have focused mainly on the biomedical aspects of combination prevention (HIV/STI testing and condom distribution). However, in keeping with the literature presented above, ??? has always been of the opinion that without targeting tailored behavioural change, biomedical interventions will have limited effects within specific community groups. This was confirmed in data collated from ???'s previous counselling, peer support and mentoring services. Here, behavioural interventions for BAME MSM showed significant improvements in positive changes in attitudes and skills around maintaining safer sex and knowledge around HIV (28).

The above is a rare example of funded work within the UK combination HIV prevention, which has not been sufficiently broad enough to address important aspects of behavioural issues, while also neglecting the role of community-level interventions in developing a more sustainable approach. The restricted effects of the biomedical interventions observed in ???'s data, and supported by HIV literature, has led to the call for a more holistic HIV prevention approach in which psychosocial and behavioural strategies targeting high-risk local communities are more strongly emphasised. As a result of their position on effective HIV prevention, there has been a shift in the ???'s focus from mass, brief interventions to more intensive and interpersonal ones. The current paper represents a collaboration between ??? and an external researcher. It underlines the assumption that further exploration is required in order to understand the effectiveness of successful behavioural strategies at local levels of interventions. This community-research collaboration follows the rationale advocated in the framework for community-university partnership. Here, the mutual desire in achieving social change (29) through the facilitating role of the catalytic researcher partner (30), provides opportunities for the organisation to increase community-based behavioural interventions' visibility through innovative academic contribution. Accordingly, the outcomes of this review will create the foundation for more effective interventions and solutions, increase both community capacity and sustainability, while reinforcing the value of community-based research.

In this respect, a comprehensive review of empirical studies was undertaken to investigate the reliability of community-based HIV prevention practices, which have targeted behavioural change in demographics and contexts similar to those presented in London. The ultimate aim would be to develop recommendations potentially directed at London's HIV and sexual health leaders, researchers, practitioners and policy makers, about strategy implementation in real-world settings.

\section{Methods}

A review was conducted utilising a combination of keywords while bearing in mind restricting inclusion criteria (language - English, geographic location Western world, and date - 2010 onwards). In order to include studies based in high-income contexts outside the USA, the search also comprised data from European studies published since 2000. Other key elements for inclusion referred to target population, methods of intervention and outcome variables. Interventions follow-up effects were limited to a maximum of 12 months. The strategy and eligibility criteria within the search process are shown in Figure 1.

\section{Results}

As a result, a final set of 22 community-based studies were included in the review. The interventions characteristics are described in Table I. All the behavioural approaches identified as part of community-based interventions considered within the analysis were implemented in urban geographical areas. Of these, 17 were from USA, 4 were based in Europe (31-34), and 1 was from Australia (35). There were 4 community-based behavioural interventions that specifically combined with elements of structural levels of prevention. This involved stigma (36), public housing and unemployment (37-39). A further 9 behavioural approaches based on community-level studies were combined with biomedical HIV-related preventative strategies such as PrEP uptake and knowledge (4042), HIV testing (35, 43-45), Antiretroviral Therapy (ART) adherence (46) and condom use (47). 
The designs of the 22 studies were a mix of randomised control trials and observational studies, comprising a total number of 6637 participants. The majority targeted MSM $(n=11)$. The remainder were based on interventions conducted with BAME $(n=9 ; 36,37,39,43,45,47-50)$, PLWHIV $(n=6 ; 36-38,46,50,51)$, heterosexuals $(n=2 ; 39,48)$, women $(n=5 ; 32,36,45,47,49)$, sex workers (SW, 49), individuals convicted of crime (ICC, 52), and finally individuals of transgender experience (ITE, $n=2 ; 38,41)$. The duration of the interventions varied. The majority $(n=14)$ were delivered in 3 to 6 sessions, with 3 separate interventions delivered respectively through 1,7 and 13 sessions. The total hours of intervention exposure ranged from 1 to 28 . In 5 studies ( $31,34,35,37,49$ ), the total number of sessions was not reported, along with further 3 interventions not having the total exposure time being disclosed $(38,44,46)$.

\section{Study Content and Successful Outcomes}

The majority of the interventions included skills building in areas such as HIV risk knowledge and understanding of sexual risk behaviours. Specifically, 11 interventions included personal skills building to reduce sexual risk behaviours using strategies such as couple-based preventative approach (52), seronegative women (33), and a mixture of individual and group interventions. Among these, DeMarco and Chan (36) used four-week-based peer-led small groups of expressing-writing workshops to share stories and emotions between PLWHIV. Furthermore, 5 interventions, 2 of which targeted a total of $n=169$ women from BAME communities $(47,49)$, included components of culturally tailored HIV-related counselling where social or group support was encouraged.

Sexual risk reduction strategies, such as number of sexual partners, condom use and negotiation of condomless sex were tackled in 10 interventions. Of these, 1 involved ICC (52), 2 included MSM $(31,34), 3$ had BAME as participants $(39,47,48)$, and finally 1 targeted PLWHIV (51). Furthermore, 7 interventions involved the development of strategies to reduce substance use, with 5 specifically targeting the MSM population. Of these, 2 studies used peer-based facilitators $(42,43)$. Finally, the 3 interventions that focussed on ART adherence targeted PLWHIV $(46,50)$ and PrEP users $(41)$. These interventions also included a sexual risk behavioural component within the delivery of both individual and group counselling approaches.

The majority of successful studies' outcomes resulted from interventions that increased condom use $(n=12)$. These results remain constant in follow-ups ranging between 6 weeks and 12 months. Two interventions targeted HIV transmission risks in PLWHIV. One significantly achieved viral suppression using an approach comprising of socio-structural elements such as stigma and housing (37). The other saw a reduction of the viral load through computer-based counselling interventions (46). Other significant intervention effects highlighted an increase in HIV knowledge in both MSM, by implementing multi-session group work addressing emotions and knowledge (33); and BAME women via peer-mentoring support through house parties for members of the same social network (49). Others showed improvements in HIV testing in MSM, while adopting on average 10-minute peer-led interventions targeting sexual health promotion on a large-scale $(n=1484)$ such as in gay bars (31). Jamil and colleagues (35) demonstrated that similar improvements were seen at 12 months follow-up resulting from interventions aimed at self-testing. Furthermore, reductions of both HIV transmission and STI were registered respectively at 12 months follow-up when using 5-session couple-based counselling (52), and 9 months follow-up after intensive integrated behavioural interventions such as individual counselling and group sessions on knowledge sharing and skills building (50). Interestingly, 5 out of 6 interventions that successfully reduced the number of sexual partners in their participants had HIV and STI testing as part of their outcome variables $(42,43,45,48,52)$. This demonstrates that the inclusion of community-based counselling as a preventative strategy along with testing could readily be perceived as a predictor of safer practices in sexual behaviours.

Furthermore, 6 interventions demonstrated a reduction in risky behaviour within vulnerable populations. With respect to BAME, Sapiano et al. (45) administered culturally relevant, gender specific 5-session of 2 hours within small group interventions. Fisher et al. (48), instead, implemented a 50-minute video opportunity promoting condom education and safer sex, while emphasising condom negotiation skills. In relation to PLWHIV, Sikkema et al. (51) adopted a 3-session brief risk reduction consisting of post-test counselling on sexual health information, disclosure decision making and risk reduction. Finally, when working with MSM, Mimiaga and colleagues (44) successfully used a 13-session intervention integrating behavioural activation, sexual risk reduction counselling and cognitive behavioural therapy to tackle substance abuse and depression. Similarly, interventions involving BAME women such as in Sanchez et al.'s (47), identified an increment on risk perception and intention for safe sex at 6 months follow-up, by using culturally tailored small group levels within a 3-week period to increase cognitive (HIV knowledge, self-efficacy through role playing and self sex negotiation) and behavioural (condom use) attitudes. Additionally, 4 interventions for ICC $(52)$, MSM $(42,44)$ and PLWHIV $(51)$ targeted and successfully reduced alcohol and drug-related risk behaviours as a result of peer intervention, individual counselling and couple-based HIV and STI preventative approaches.

Other significant outcomes due to community-based behavioural interventions identified an increased desire to use PrEP, its adherence and knowledge. For instance, Desrosiers et al. (40) used a person-centred care coordination approach based on human motivation theory, wherein young Black MSM received personalised comprehensive PrEP education, sexual risk reduction counselling, while also exploring perceived barriers in accessing PrEP. Similarly, Liu et al. (41) adopted 15-minute individual-centred risk reduction and adherence counselling, where MSM/ITE participants discussed sexual activities, substance use, barriers to PrEP pill-taking and strategies to promote adherence. Further interventions for heterosexual Black men (39) positively linked preventative behavioural interventions on HIV risk reduction to structural risks of housing and employment, using 3 60-minute sessions including 1-2 brief 10-minute checkin meetings in between. Gollub et al.'s intervention (32) increased knowledge about protection at a 5-week follow up, while implementing a weekly-based 5session group counselling and conscious raising to educate women from diverse cultural backgrounds about body empowerment and sex risk reduction. Finally, Martinez and colleagues (38) increased participation of high-risk population groups such as PLWHIV and individuals of transgender experience through peer-led 6-session stigma reduction hybrid individual- and group-based interventions. This comprised the use of cognitive behavioural techniques and role playing to maintain health while reducing HIV/STI risks.

\section{Discussion}

The current paper underlined the assumption that real efforts in using community-based behavioural interventions as part of combination prevention in London should be increased, while carefully considering the dynamic network of dimensions located both at the individual and contextual levels. Given that in 
this paper individuals' behavioural change was considered to be the primary means of achieving the goal of getting London to zero infections, a review was undertaken to explore the interventions that have previously been used to reduce HIV infections at community levels in similar contexts worldwide.

\section{Evidence Supporting the Efficacy of Community-based Behavioural Interventions Focused on Combination HIV Prevention}

The review highlights the overall effectiveness of behavioural strategies within community-based projects using counselling (individual and group levels), peer support mentoring intervention and couple-focused approach, either face-to-face or remotely (video or computer-based). Successful approaches mostly targeted at-risk population groups disproportionately affected by the epidemic and in need of effective preventative strategies. The use of behavioural modification training within local communities in enhancing safe sex was considered to be effective. Specifically, it improved HIV risk knowledge, limited the number of sexual partners, and increased both condom use and the negotiation of unprotected sex. Furthermore, the review highlighted an increase in both HIV testing and ART adherence. Moreover, it showed improvements in HIV viral suppression and reductions of both HIV and STIs. This demonstrates that community-based approaches are successful in achieving early diagnoses and access to ART, leading to the reduction of HIV transmission.

Other promising findings shown throughout the review referred to the enhancement of PrEP desire, adherence and knowledge as a result of behavioural interventions at community levels. These mirror the case studies' outcomes referred to in the introduction, where effective London-based community-level interventions (??? PrEP Champions Project, 11; ???'s PrEP exploratory research, 12) were shown to increase knowledge and accessibility of PrEP in vulnerable groups. Specifically, the evidence resulting from both the review and the London-based interventions demonstrate how knowledge and accessibility are complementary in tackling the problem of stigma as the major barrier to accessing PrEP. In fact, it is because of the stigma attached to PrEP use that people fear being judged negatively by their community members. Other successful interventions designed to operate at community levels when targeting structural elements such as stigma included opinion leaders. These showed to be powerful in changing social norms, and subsequently, in influencing individual behaviours in relation to HIV prevention within a specific context. Here, counselling and peer support mentoring interventions have brought significant improvements in attitudes and skills in order to maintain safer sex.

This aspect thus reinforces the idea that individuals are able to gain autonomy over their sexual behaviours once becoming aware of risks and their own choices. We argue here that in the absence of behavioural interventions that prevent stigma from hampering knowledge and awareness at community levels, HIV prevention biomedical tools would have restricted impact. Therefore, for the widespread use of any HIV-related medication, it is important that individuals at risk feel emotionally safe to be well informed about its benefits in order to make informed choices (24). The review found that increasing knowledge about HIV transmission and its strategies for prevention helped the studies' participants perceive their level of risk of infection. Here, HIV-related awareness enhanced the reduction of risky behaviour and strengthened the knowledge necessary to enable individuals to protect themselves from potential transmission. This is a very important aspect, since past research (9) has shown that lack of risk perception can limit acceptability of the concept of TasP. The idea that incorporating skills-building behavioural interventions and stigma-related structural interventions within any community-based HIV preventative programme is fundamental. As such, this paper agrees with Flowers and colleagues (13) when considering behavioural interventions to be supporters rather than competitors of biomedical technologies.

With regard to the importance of providing the combination of multi-level interventions, the review also illustrated the importance of both structural and community-based approaches in order to increase effectiveness within high-risk environments. This was particularly so in contexts where endangered structural factors (homelessness and unemployment) could easily magnify the likelihood of infections. The above demonstrates that interventions should always be contextually appropriate and specific to their audience's values and needs when attempting to change behaviours. As a result, we argue that communities should function as primary agents in any local prevention efforts, where community-based actors are identified as intervention specialists (16). In this respect, they should be actively involved in the development, implementation, and oversight of programme-interventions intended to change individuals' behaviours. One of the rationales behind this is to understand that increasing knowledge of HIV in the community, while also respecting the human rights of those targeted, is critical in reaching the marginalised groups with effective HIV prevention services. Community ownership would therefore enable open discussions on community knowledge and perspectives of HIV and facilitate the engagement of those at risk, while increasing the chances for successful interventions.

Within the review, there was found to be a paucity in terms of community-based studies from the UK in the past ten years. This means that evidence-based community-based behavioural interventions have not been implemented, published or reported consistently. This should be perceived as a loss of opportunity for the advocacy of community-based behavioural interventions in the UK context, particularly when considering the limiting effects of the over-reliance on biomedical tools (13). As Collins and colleagues (17) have previously asserted, evidence-based behavioural strategies within community-level interventions have the power to bridge the gap between communities at risk and HIV prevention intervention research. This concern cannot be overestimated when aiming to end AIDS by 2030. Therefore, further efforts should be directed towards documenting and evaluating such interventions.

Finally, the majority of the studies used combination HIV prevention as part of their strategies of intervention. This means that the behavioural aspects were combined with biological and structural elements of prevention. The current paper considers this to be a positive sign, while agreeing with Noar (21) when highlighting the importance of behavioural approaches within community-based interventions in supporting recent advances of biomedical strategies. In order to achieve optimal public health impact in the real-world context, simultaneous implementation of both HIV treatment and prevention plays a vital role when used within the comprehensive behavioural, biomedical and structural combination of strategies adaptable to a specific setting. Unfortunately, from this point of view it seems that the combination HIV prevention model in the UK still has a long way to go.

\section{RECOMMENDATIONS}

We argue that in the absence of community-based culturally tailored behaviour change interventions, HIV prevention relying on a solely biomedical approach would have a restricted impact in helping London to get to zero new HIV infections. Consequently, after summarising the review and examining emerging 
issues, a number of recommendations are proposed at different levels, with the further need to expand a more robust collaboration between community-based interventions and empirical research. Through this, it is hoped that the effectiveness of these behavioural interventions will increase respect within the multidimensional combination of HIV prevention in London.

\section{Recommendations at the Individual Level}

Evidence from the UK context shows an increased practice of condomless anal sex in MSM and a scarcity of prevention interventions directed at women, requiring additional effort to be placed on counselling and peer mentoring. The review has illustrated the positive aspects of using both video and peer-based facilitators to promote safer sex and increase HIV knowledge in MSM. These strategies could be also replicated with MSM who are reluctant to test for HIV, particularly as small group-level interventions in targeted familiar environments (e.g., bars or other LGBT+ venues). Pre- and post- peer-based counselling will facilitate access to testing, while discussing with service users any potential barriers to the use of condoms. Peer mentoring becomes a valid alternative to help create an empathic approach as this presents the ability to understand the emotional burdens that similar individuals face in relation to protection, HIV testing, diagnosis and treatment.

Further recommendations should aim to reach out to women, particularly those from minority backgrounds. For example, evidence from the review has shown that culturally tailored HIV-related counselling and group support are effective interventions in seronegative women from ethnic minority background. Specifically, the review has demonstrated the effectiveness of adopting emotion-based writing workshops and computer-based counselling in combination with structural elements such as stigma. Consequently, it is strongly believed that a community coaching model using groups in targeting stigma-related issues could assist women at risk on how to best approach both self-sampling and self-testing without the risk of feeling discriminated. Community-level distribution of test kits together with contextually tailored behavioural interventions, particularly for women from the Black African community is strongly recommended. In this instance, community-based counselling through established support groups would help women to understand the importance of testing or safer sex and receive both health and psychological back up. This method of intervention will be community-based in the sense that specific settings will be targeted as spaces to facilitate the intervention: home-base networking, spiritual and religious sites, communal gathering venues such as hairdressers and other familiar publicly frequented spaces. The goal of promoting a supportive and enabling environment for women will provide the opportunity to address through community dialogue the underlying inequalities faced by this high-risk population group.

\section{Recommendations at the Community Level}

Behavioural interventions also aim at building active connected communities through constructed dialogue. Giving voice to members of high-risk groups create conditions for them to play a more active role in community life. The review has shown that interventions implementing group counselling and awareness raising have been successful in empowering individuals from diverse cultural backgrounds, while enhancing sex risk reduction. However, these interventions also have the ability to inform and educate, while providing communities with the relevant information tailored to the needs of their local contexts. This includes information regarding the benefits of testing, how to access HIV testing services, the importance of testing as a communal responsible act, and the benefits of knowing their own status. In cities with a richness of cultures and languages, such as London, particular attention must be given to any non-English speaking subgroups. Here, culturally sensitive information needs to be translated appropriately while considering the meaning, nuances and cultural specificity of the messaging. The aim is to tackle the physical, social and legal realities within which unsafe attitudes take place, wherein group counselling and peer mentoring activities are included in culturally appropriate interventions.

\section{Recommendations at the Structural Level}

HIV authorities in England should attempt to alter social norms by including policy interventions that produce behavioural change. Here, structural elements such as inequality and stigma should always be considered. It is recommended that interventions should include a combination of policy actions and other programmatic initiatives that would target gender, economic and social inequality. These should also include antidiscrimination laws that support human rights. Further national strategies should see HIV prevention as a continuum. Here, the funding of comprehensive programmes should include a coordinated system of technical support in developing an inclusive HIV-related national plan. Its objective is to increase awareness of STI and HIV prevention strategies and treatment options, while also aiming at reducing levels of stigma within affected communities.

\section{Recommendations for Improved Evaluation of Community-based Interventions}

Finally, from the review it was evident that inconsistent implementation of evidence-informed interventions caused limitations in some of the studies when transferred to a different context. It is strongly believed that community-based behavioural interventions are well suited to improve health, including HIV prevention. However, the evidence of its effectiveness cannot be objectively evaluated without reliable outcome documentation. At present, most communitybased service-delivery in London has been documented through grey literature, with very little evaluation made through robust studies. Therefore, conducting more robust evaluations in order to identify community-level best practices for health improvement is highly recommended.

A valid alternative would be to establish a framework for conceptualising community-academic partnerships around capacity building (53). This advocates for community-based organisations to improve their evaluation-based capacity, fundamental in the collection, analysis and interpretation of own data in order to make sense of their actions and improve results. This means that in order to maximise the accuracy of information about a specific intervention, serviceproviders should receive training on how the data should be collected and evaluated. Quality of information through the provision of robust evaluation and monitoring will be fundamental in assessing the effectiveness (including evaluation of costs) of a specific intervention. Through this, it could be possible to identify key behaviours, context-related values and optimal delivery strategies, while giving greater emphasis to understanding the key trends of local epidemics. This strengthened monitoring and evaluation capacity will allow for the impact and value of any HIV preventative programmes to be effectively 
assessed, while also informing decisions on further allocation of funding. The final goal would be a system that comprises use of intervention protocols, technical assistance, training, and fidelity assessment.

At present, the system appears to operate in a vicious cycle of budgetary constraints that national HIV prevention programmes have faced in these past years (54). By not funding the provision of evidence, it is impossible for community-based behavioural interventions to show reliable empirical conclusions. At the same time, the inability to establish the interventions' effectiveness (including cost-effectiveness) limits the reliability of these approaches and therefore their subsequent funding. This means that lack of community embedded evidence becomes a self-fulfilling prophecy. Increasing emphasis on evidence-based community behavioural interventions will contribute to the efforts in understanding the key trends of local epidemics, and assess the overall feasibility of successful behavioural programmes into the London context.

\section{Limitations}

Various limitations should be also considered when analysing this paper. Only studies from USA where found to be undertaken after the year 2010 , wondering whether these could be easily transferred to the UK context. Consequently, in order to have studies based in high-income contexts outside the USA, these were further included by expanding the scope of publication back to 2000 . The reviewed studies' interventions follow-up effects were limited to a maximum of 12 months, despite previous research specifically showing that long-term ( $>1$ year) changes are difficult to sustain (55). This is an issue requiring further investigation. The reviewed studies over-relied mostly on self-reported or less robust assessment, rather than complementing with more objectively measured behavioural interventions (e.g., randomised controlled trials). Finally, the lack of detailed interventions' descriptions within the studies' reports restricted the objectivity in assessing the effectiveness of their interventions. A weakness that further community-based behavioural interventions research should consider when reporting their outcomes in the future.

\section{Conclusions}

The current paper outlines some guiding principles and considerations that policy makers in London must bear in mind when considering the effectiveness of combination HIV prevention with the aim of reaching zero HIV infections by 2030 (Summary of the study outcomes, description and recommendations are shown in Table II). To achieve this, it is fundamental that biomedical and structural elements of prevention are supported by evidence-based behavioural interventions in partnership with community-based HIV prevention providers. This would direct the action of national stakeholders in implementing evidencebased interventions, which should be in line with the epidemiological data of a specific context and supported by social and structural political commitment. This continuum of prevention would then be rigorously evaluated, adapted and further implemented through the systematic analysis of research data. The authors of this paper are aware that this entire process presents some challenges. However, it is also believed that these challenges are not insurmountable. The encouragement of a participative ethos and of multilevel interventions, both at community and research levels, can only improve the health situation and outlook of vulnerable communities affected by HIV in London. The findings of this review have reinforced our argument that we need community-based, culturally relevant programmes alongside and interweaving with biomedical approaches, and not solely biomedical-focused HIV prevention.

\section{Declarations}

Funding: The authors have no relevant financial or non-financial interests to disclose and have no competing interests to declare that are relevant to the content of this manuscript.

Conflicts of interest/Competing interests (Not Applicable)

Ethics approval (Not Applicable)

Consent to participate (Not Applicable)

Consent for publication (Not Applicable)

Availability of data and material (Not Applicable)

Code availability (Not Applicable)

\section{References}

1. Joint United Nations Programme on HIV/AIDS (UNAIDS). Getting to zero: 2011-2015 strategy Joint United Nations Programme on HIV/AIDS (UNAIDS) (UNAIDS/10.12E / JC2034E (English original, December 2010). Available at: https://unaids test.unaids.org/sites/default/files/unaids/contentassets/documents/unaidspublication/2010/20101221_JC2034E_UNAIDS-Strategy_en.pdf.

2. Holmes KK, Levine R, Weaver M. Effectiveness of condoms in preventing sexually transmitted infections. Bull World Health Organ. 2004;82(6):454-61.

3. Frieden TR. A framework for public health action: The health impact pyramid. Am J Public Health. 2010;100(4):590-5.

4. Nash S, Desai S, Croxford L, et al. Progress towards ending the HIV epidemic in the United Kingdom: 2018 report (issued in November). London: Public Health England. Available at:

https://assets.publishing.service.gov.uk/government/uploads/system/uploads/attachment_data/file/821273/Progress_towards_ending_the_HIV_epidem 
5. Logan L, Fakoya I, Howarth A, et al. Combination prevention and HIV: a cross-sectional community survey of gay and bisexual men in London, October to December 2016. Eurosurveillance. 2019;24(25):1-8.

6. Aghaizu A, Brown AE, Nardone A, Gill ON. Delpech, V. HIV in the United Kingdom (2013 Report). London: Public Health England; 2013.

7. British HIVA. British HIV Association guidelines for the routine investigation and monitoring of adult HIV-1-positive individuals 2016 (BHIVA). Available at: .

8. Sophia Forum and Terrence Higgins Trust. Women. and HIV: Invisible No Longer. A national study of women's experiences of HIV. Available at: https://www.tht.org.uk/sites/default/files/2018-08/women-and-HIV_report_final_amended.pdf.

9. Young I, Flowers P, McDaid LM. Key factors in the acceptability of treatment as prevention (TasP) in Scotland: a qualitative study with communities affected by HIV. Sex Transm Infect. 2014;91(4):269-74.

10. World Health Organization. Consolidated guidelines on the use of antiretroviral drugs for treating and preventing HIV infection: recommendations for a public health approach. 2nd ed. Geneva: World Health Organization; 2016.

11. West R, Dunkley Y, Wang D, et al. Attitudes and factors to PrEP uptake among HIV risk groups across London. 25th BHIVA Annual Conference 2019, Bournemouth, UK. HIV Medicine. 20(5):13.

12. Di Giuseppe K, Kasoka K, Dunkley Y. Investigating preexposure prophylaxis (PrEP) uptake among Black African communities in East London and Hertfordshire: A brief report of a mixed methods Study. International Journal of Sexual Health. 2019;31(3):291-6.

13. Flowers $\mathrm{P}, \mathrm{Wu} \mathrm{O}$, Lorimer $\mathrm{K}$, et al. The clinical effectiveness of individual behaviour change interventions to reduce risky sexual behaviour after a negative human immunodeficiency virus test in men who have sex with men: systematic and realist reviews and intervention development. Health Technol Assess. 2017;21(5):1-164.

14. Choi SKY, Holtgrave DR, Bacon J, et al. Economic Evaluation of Community-Based HIV Prevention Programs in Ontario: Evidence of effectiveness in reducing HIV infections and health care costs. AIDS Behav. 2015;20(6):1143-56.

15. Charania MR, Crepaz N, Guenther-Gray C, et al. Efficacy of structural-level condom distribution interventions: A meta-analysis of U.S. and international studies, 1998-2007. AIDS Behav. 2010;15(7):1283-97.

16. Ross MW, Williams ML. Effective targeted and community HIV/STD prevention programs. J Sex Res. 2002;39(1):58-62.

17. Collins C, Harshbarger C, Sawyer R, Hamdallah M. The diffusion of effective behavioral interventions project: Development, implementation, and lessons learned. AIDS Educ Prev. 2006;18(4A):5-20.

18. Forde J, Kirwan P, Louth R, Corkin H, Crook P. Annual epidemiological spotlight on HIV in London - 2017 data (issued in December). London: Public Health England. 2018. Available at:

https://assets.publishing.service.gov.uk/government/uploads/system/uploads/attachment_data/file/767460/LondonHIVSpotlight2017.pdf.

19. Suthar AB, Ford N, Bachanas PJ, Wong VJ, Rajan JS, Saltzman AK, Ajose O, Fakoya AO, Granich RM, Negussie EK, Baggaley RC. Towards universal voluntary HIV testing and counselling: A systematic review and meta-analysis of community-based approaches. PLoS Medicine. 2013;10(8):e1001496. https://doi.org/10.1371/journal.pmed.1001496.

20. Herbst JH, Beeker C, MathewA, et al. The Effectiveness of Individual-, Group-, and Community-Level HIV Behavioral Risk-Reduction Interventions for Adult Men Who Have Sex with Men. Am J Prev Med. 2007;32(4S):38-67.

21. Noar SM. Behavioural interventions to reduce HIV-related sexual risk behaviour: Review and synthesis of meta-analytic evidence. AIDS Behav. 2008;12:335-53.

22. Elford J, Sherr L, Bolding G, Serle F, Maguire M. Peer-led HIV prevention among gay men in London: Process evaluation. AIDS Care. 2002;14(3):351-60.

23. Ross DA. (2010). Behavioural interventions to reduce HIV risk: what works? AIDS. 2020; 24(4):S4-S14.

24. Global HIV Prevention Working Group (GHPWG). Behaviour change and HIV prevention: (Re)considerations for the 21st century (issued in August). 2008. Available at: http://www.globalhivprevention.org/pdfs/PWG_behavior\%20report_FINAL.pdf.

25. Albarracín D, Gillette JC, Earl AN, Glasman LR, Durantini MR, Ho MH. A test of major assumptions about behavior change: A comprehensive look at the effects of passive and active HIV-prevention interventions since the beginning of the epidemic. Psychol Bull. 2005;131(6):856-97.

26. Burton J, Darbes LA, Operario D. Couples-focused behavioral interventions for prevention of HIV: Systematic review of the state of evidence. AIDS Behav. 2008;14(1):1-10.

27. Lyles CM, Kay LS, Crepaz N, et al. Best-Evidence interventions: Findings from a systematic review of HIV behavioral interventions for US populations at high risk, 2000-2004. Am J Public Health. 2007;97(1):133-43.

28. Wardle R, Wang D. GMI Partnership counselling and mentoring report: Quarter 4 Jan-Mar (2013-2014). Pan London HIV Prevention Programme. Unpublished report. 2014.

29. Burlew A, Shambley-Ebron D, Lanaway D, McCuistian C, Sherman L, Steele L. Community collaborative cultural adaptation: creating culturally appropriate behavioral interventions through university and community collaborations. Collaborations: A Journal of Community-Based Research Practice. 2018;2(1):1.

30. Milofsky C. The catalyst process: What academics provide to practitioners. Nonprofit Management Leadership. 2006;16(4):467-80.

31. Flowers P, Hart GJ, Williamson LM, Frankis JS, Der GJ. Does bar-based, peer-led sexual health promotion have a community-level effect amongst gay men in Scotland? Int J STD AIDS. 2002;13(2):102-8.

32. Gollub EL, Brown EL, Savouillan M, Waterlot J, Coruble G. A community-based safer-sex intervention for women: Results of a pilot study in south-eastern France. Culture Health Sexuality. 2002;4(1):21-41. 
33. Harding R, Bensley J, Corrigan N, et al. Outcomes and lessons from a pilot RCT of a community-based HIV prevention multi-session group intervention for gay men. AIDS Care. 2004;16(5):581-5.

34. Imrie J. A cognitive behavioural intervention to reduce sexually transmitted infections among gay men: randomised trial. BMJ. 2001;322(7300):1451-6.

35. Jamil MS, Prestage G, Fairley CK, et al. Effect of availability of HIV self-testing on HIV testing frequency in gay and bisexual men at high risk of infection (FORTH): a waiting-list randomised controlled trial. The Lancet HIV. 2017;4(6):e241-50.

36. DeMarco RF, Chan K. The Sistah Powah structured writing intervention: A feasibility study for aging, low-income, HIV-positive black women. Am J Health Promot. 2013;28(2):108-18.

37. Brewer R, Daunis C, Ebaady S, et al. Implementation of a socio-structural demonstration project to improve HIV outcomes among young Black men in the deep South. J Racial Ethn Health Disparities. 2019;6(4):775-89.

38. Martinez O, Lopez N, Woodard T, Rodriguez-Madera S, Icard L. Transhealth information project: A Peer-Led HIV prevention intervention to promote HIV protection for individuals of transgender experience. Health Soc Work. 2019;44(2):104-12.

39. Raj A, Dasgupta A, Goldson I, Lafontant D, Freeman E, Silverman JG. Pilot evaluation of the Making Employment Needs [MEN] Count intervention: Addressing behavioral and structural HIV risks in heterosexual Black men. AIDS Care. 2013;26(2):152-9.

40. Desrosiers A, Levy M, Dright A, et al. A randomized controlled pilot study of a culturally-tailored counseling intervention to increase uptake of HIV preexposure prophylaxis among young black men who have sex with men in Washington, DC. AIDS Behav. 2018;23(1):105-15.

41. Liu AY, Cohen SE, Vittinghoff E, Anderson PL, Doblecki-Lewis S, Bacon O. HIV pre-exposure prophylaxis integrated with municipal and community based sexual health services. JAMA Intern Med. 2016;176(1):75-84.

42. Mimiaga MJ, Hughto JMW, Reisner SL. A randomized pilot study of a Group-Delivered HIV risk reduction intervention for At-Risk urban men who have sex with men who regularly attend private sex events. Arch Sex Behav. 2019;48(4):1059-71.

43. Hosek SG, Lemos D, Hotton AL, et al. An HIV intervention tailored for black young men who have sex with men in the House Ball Community. AIDS Care. 2014;27(3):355-62.

44. Mimiaga MJ, Pantalone DW, Biello KB, et al. An initial randomized controlled trial of behavioral activation for treatment of concurrent crystal methamphetamine dependence and sexual risk for HIV acquisition among men who have sex with men. AIDS Care. 2019;31(9):1083-95.

45. Sapiano TN, Moore A, Kalayil EJ, et al. Evaluation of an HIV prevention intervention designed for african american women: Results from the SISTA Community-Based organization behavioral outcomes project. AIDS Behav. 2012;17(3):1052-67.

46. Kurth AE, Spielberg F, Cleland, et al. Computerized counseling reduces HIV-1 viral load and sexual transmission risk. J Acquir Immune Defic Syndr. 2014;65(5):611-20.

47. Sanchez M, Rojas P, Li T, et al. Evaluating a culturally tailored HIV risk reduction intervention among latina immigrants in the farmworker community. World Med Health Policy. 2016;8(3):245-62.

48. Fisher HH, Patel-Larson A, Green K, et al. Evaluation of an HIV prevention intervention for African Americans and Hispanics: Findings from the VOICES/VOCES community-based organization behavioral outcomes project. AIDS Behav. 2011;15(8):1691-706.

49. Hawk M. The Girlfriends Project: Results of a Pilot Study Assessing Feasibility of an HIV Testing and Risk Reduction Intervention Developed, Implemented, and Evaluated in Community Settings. AIDS Educ Prev. 2013;25(6):519-34.

50. Kalichman SC, Cherry C, Kalichman MO, et al. Integrated behavioral intervention to improve HIV/AIDS treatment adherence and reduce HIV transmission. Am J Public Health. 2011;101(3):531-8.

51. Sikkema KJ, Hansen NB, Kochman A, et al. The development and feasibility of a brief risk reduction intervention for newly HIV-diagnosed men who have sex with men. J Community Psychol. 2011;39(6):717-32.

52. El-Bassel N, Gilbert L, Goddard-Eckrich D, et al. Effectiveness of a couple-based HIV and sexually transmitted infection prevention intervention for men in community supervision programs and their female sexual partners. JAMA Netw Open. 2019;2(3):e191139.

53. Hogan KS, Tynan JM, Covill VJ, Kilmer RP, Cook JR. A capacity building framework for community-university partnerships. Collaborations: A Journal of Community-Based Research and Practice. 2017; 1(1). Available at: http://scholarlyrepository.miami.edu/collaborations/vol1/iss1/1.

54. White C. Sexual health services on the brink. BMJ (Clinical research ed.). 2017; 359, j5395.

55. Coates TJ, Richter L, Caceres C. Behavioural strategies to reduce HIV transmission: how to make them work better. The Lancet. 2008;372(9639):669-84.

\section{Tables}

Table 1 can be found in the supplementary files section. 
Table II

Summary of the study outcomes, description and recommendations

$\begin{array}{lll}\text { Study } & \text { Outcome Description } & \text { Recommendations } \\ \text { Outcomes } & \end{array}$

Overall Behavioural strategies within community-based using counselling (individual and group

Effectiveness levels), peer support mentoring, couple-focused approaches, video or computer-based interventions are effective in targeting vulnerable population groups: BAME, MSM, PLWHIV, IDU, ICC, SW and ITE.

Knowledge \& Behavioural strategies within community-based enhance safe sex and HIV risk knowledge Accessibility limit the number of sexual partners, increase condom use and negotiation of unprotected intercourse, increase HIV testing and ART adherence, improve HIV viral suppression while reducing HIV and STIs infections, and finally enhance PrEP desire, adherence and knowledge.

Combination of Interventions designed to operate at community levels are successful when targeting Structural structural elements such as stigma. These are powerful in changing social norms and Elements influencing individual behaviours in relation to HIV prevention: Opinion leaders, counselling and peer support mentoring interventions bring significant improvements in attitudes and skills in order to maintain safer sex.

Such interventions are vital elements within the combination HIV prevention for high-risk communities.

Knowledge and accessibility are complementary in tackling the problem of stigma as the major barrier to accessing HIV prevention.

In the absence of behavioural interventions that prevent stigma from hampering awareness, HIV prevention biomedical tools would have restricted impact.

$\mathrm{HIH}$-related Increasing knowledge about HIV transmission and its strategies for prevention helps to Awareness perceive individuals' level of risk for infection. Here, HIV-related awareness enhances the reduction of risky behaviour and strengthened the knowledge necessary to enable individuals to protect themselves from potential transmission.

Individuals are able to control their own sexual behavioural patterns mainly once becoming aware of risks.

Multi-level Importance of both structural and community-based approaches in order to increase Intervention effectiveness within high-risk environments, especially in contexts where endangered structural factors (homelessness and unemployment) could easily magnify the likelihood of infections.

Interventions should always be contextually appropriate, and that community actors function as primary agents in any local prevention efforts in reaching the marginalised groups.

Lack of There is a paucity in terms of community-based studies from the UK in the past ten years. Empirical This means that evidence-based community-based behavioural interventions have not

Evidence been implemented, published nor reported consistently.

Further efforts should be directed towards documenting and evaluating such interventions in understanding the key trends of local epidemics.

Implementation Inconsistent implementation of evidence-informed interventions caused limitations in \& Sustainability some of the studies when transferred to a different context, and only few studies measured the outcomes with follow-up periods up to 12 months.

Short-term follow-ups prevent service providers from examining longer-term efficacy and sustainability of intervention effects.

\section{Figures}




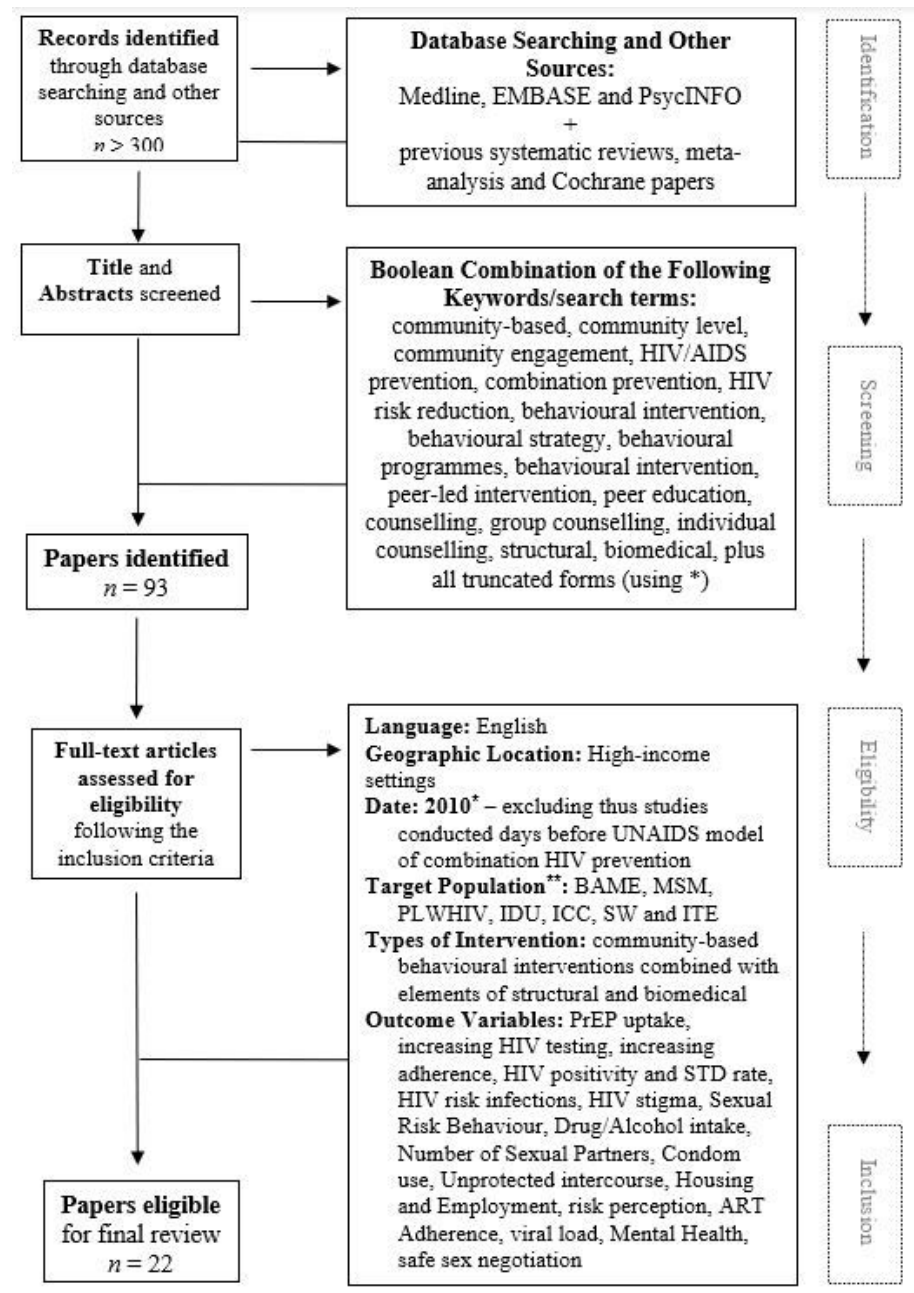

Figure 1

Diagram representing the process of identification, screening, eligibility and inclusion of the community-based behavioural interventions' effectiveness for final review.

* In order to include studies based in high-income contexts outside the USA, the search also comprised data from European studies published back to 2000.

${ }^{* \star}$ BAME = Black African and Minority Ethnic, MSM = Men who have Sex with Men, PLWHIV = People Living with HIV, IDU = Injecting Drug Users, ICC = Individuals Convicted of Crime, SW = Sex Workers, ITE = Individual of Transgender Experience.

\section{Supplementary Files}

This is a list of supplementary files associated with this preprint. Click to download.

- Tablel.docx 